\title{
VALIDADE E FIDELIDADE DA VERSÃO PORTUGUESA REDUZIDA DO WEB BASED LEARNING ENVIRONMENT INVENTORY
}

\author{
(VALIDITY AND RELIABILITY OF THE REDUCED PORTUGUESE VERSION OF THE WEB BASED \\ LEARNING ENVIRONMENT INVENTORY)
}

\author{
Angelo Jesus \\ Maria João Gomes \\ Universidade do Minho (Portugal) \\ Agostinho Cunha \\ Agostinho Cruz \\ Instituto Politécnico do Porto (Portugal)
}

\section{RESUMO}

A utilização de instrumentos padronizados de avaliação, traz consigo uma dimensão de fidelidade e validade estatística que permitem um outro olhar sobre diversos aspectos relacionados com a avaliação de ambientes de aprendizagem, complementando e enriquecendo as abordagens de carácter mais qualitativo. Nesse sentido, têm sido desenvolvidos e validados em vários países, inquéritos por questionário, com o objectivo de recolher informações para avaliação dos ambientes virtuais de aprendizagem. Em pesquisas anteriores foi já debatida a inexistência de um instrumento desta natureza, validado na língua portuguesa. Este estudo pretende colmatar esta lacuna, sendo apresentado em detalhe o processo de tradução, adaptação e validação da versão reduzida do Web Based Learning Environment Inventory.

Palavras-chave: computadores na educação, educação a distância, ambientes virtuais de aprendizagem, web based learning environment inventory.

\begin{abstract}
The use of standardized assessment instruments, brings a dimension of reliability and validity that allows a different view on various aspects related to the assessment of learning environments, complementing and enriching the more qualitative character of other approaches. In this topic, questionnaire surveys, have been developed and validated in several countries, with the aim of collecting information for evaluation of virtual learning environments. It has already been discussed, in previous works, the absence of such an instrument, validated for the Portuguese language. This study aims to fill this gap, presenting
\end{abstract}


in detail the process of translation, adaptation and validation of the Portuguese reduced version of the Web Based Learning Environment Inventory.

Keywords: computers in education, distance education, virtual learning environment, web based learning environment inventory.

As potencialidades de exploração das Tecnologias da Informação e Comunicação (TIC) no ensino superior são múltiplas, quer em termos de contextos de utilização, quer em termos de objectivos subjacentes a essa mesma exploração. Gomes (2005) sistematiza as principais vertentes e contextos de utilização das TIC no ensino, considerando que estas permitem: (1) apoiar o ensino presencial em sala de aula; (2) proporcionar oportunidades de auto-estudo com base em documentos electrónicos; (3) criar condições para o desenvolvimento de sistemas de formação a distância, (4) permitir a "extensão virtual" da sala de aula presencial e, nas suas vertentes mais centradas nas redes de comunicação, particularmente a Internet, (5) dar origem a novas modalidades de formação online que inclui na designação de e-learning. Neste cenário de integração das TIC para o desenvolvimento de aplicações educacionais, os AVA têm ganho importância como meio de mediação e gestão da educação a distância (Filho e Machado, 2002). Contudo, a expansão das formações a distância não é acompanhada por uma avaliação dessas iniciativas. O relatório do Word Bank Institute (Valcke e Leeuw, 2000) destaca que o número de avaliações de cursos e seminários em formato e-learning é limitado, sendo ainda mais reduzido nos países em desenvolvimento. Dyson e Campello (2003) argumentam que as restrições de tempo e a ausência de um conhecimento especializado são factores impeditivos para que a maioria dos promotores de iniciativas de educação a distância empreenda estudos mais detalhados de avaliação. Com a crescente integração de formações online no ensino superior universitário (Gomes, 2008) assim como na formação e educação de adultos (Filho e Machado, 2002), há uma forte necessidade de orientações e recomendações práticas para facilitar o desenvolvimento e implementação de ambientes virtuais de aprendizagem. Investigadores e docentes devem dispor de meios que permitam uma avaliação concreta e quantitativa destes ambientes virtuais, contribuindo assim para um processo contínuo de melhoria.

A avaliação dos ambientes virtuais de aprendizagem, pode ser definida como a aplicação sistemática de procedimentos para determinar, a relevância, a efetividade e o impacto de determinadas atividades, a partir dos objetivos propostos e com base em critérios pré-definidos (Laguardia, Portela e Vasconcellos, 2007). Neste sentido a avaliação não deve apenas ficar restrita à tecnologia, mas deve estenderse à interacção entre os AVA e os utilizadores no processamento da informação num determinado contexto social, pois essa interacção determina como ocorre a 
incorporação das tecnologias nos processos de ensino-aprendizagem (Ammenwerth, Gräber, Herrmann, Bürkle e König, 2003). No contexto da presente investigação, e com base nos pressupostos anteriores, pretende-se que a avaliação de AVA possa tomar como base as condições em que a aprendizagem se realiza, os modos pelos quais os estudantes são capazes de interagir sendo apoiados nas suas atividades e o alcance dos objetivos e das metas propostas. Formas qualitativas de avaliação de AVA têm sido comummente usadas por investigadores para reunir informações sobre os AVA (Tobin e Fraser, 1998), nomeadamente através de observação, os estudos etnográficos, as entrevistas e estudos de caso. Contudo, a utilização de instrumentos padronizados de avaliação, traz consigo uma outra dimensão de fidelidade e validade estatística que permitem um olhar adicional sobre diversos aspectos relacionados com a avaliação de ambientes de aprendizagem, complementando e enriquecendo as abordagens de carácter mais qualitativo. Nesse sentido, têm sido desenvolvidos e validados em vários países inquéritos por questionário, com o objectivo de recolher informações para avaliação dos ambientes virtuais de aprendizagem. Estudos prévios (Jesus, Cruz e Gomes, 2011) debateram já esta problemática e alertaram para a inexistência de um instrumento desta natureza, validado na língua portuguesa.

\section{AMBIENTES VIRTUAIS DE APRENDIZAGEM}

O conceito de ambiente virtual de aprendizagem (AVA) é geralmente entendido como um ambiente baseado na Web, utilizado para apoiar o processo de ensino e aprendizagem, na educação presencial ou a distância. Na literatura, encontramse algumas definições para AVA que vão desde um entendimento exclusivamente técnico a uma concepção mais pedagógica. Segundo Vavassori e Raabe (2003) um AVA deve reunir uma série de recursos e ferramentas, permitindo e potencializando a sua utilização em actividades de ensino-aprendizagem através da Internet, em formações a distância. Outros autores como Almeida e Almeida (2003) ou Valentini e Soares (2010) associam os AVA ao desenvolvimento de condições, estratégias e intervenções de aprendizagem num espaço virtual, de forma que seja propiciada a construção de conceitos, por meio da interacção entre alunos, professores e o objecto de conhecimento. Embora mais comummente associados à educação a distância, os AVA podem ser amplamente utilizados como suporte à aprendizagem presencial ${ }^{1}$. Ramos et al. (2009) defendem que os ambientes virtuais mais eficazes são aqueles que apresentam um conjunto integrado de recursos de comunicação e de apoio aos utilizadores, nos quais os alunos podem interagir uns com os outros, e que se inspiram numa abordagem construtivista de conhecimento. Nesse sentido, não podemos limitar o conceito de ambiente virtual de aprendizagem, à mera estrutura fornecida por um Learning Management System (LMS), mas ter presente 
e considerar a influência fundamental do modelo de organização e da abordagem pedagógica desenvolvida pelo docente. Embora os registos de dados e os relatórios de acesso fornecidos pelos LMS possam constituir indicadores do envolvimento dos estudantes com o conteúdo disponibilizado, não se pode assumir que a simples exposição do estudante ao conteúdo, por si só assegura a aprendizagem do mesmo. Outros factores, decorrentes do desenho instrucional e das estratégias pedagógicas adoptadas pelos docentes, podem ter um papel determinante nesse processo.

\section{AVALIAÇÃO DE AMBIENTES VIRTUAIS DE APRENDIZAGEM}

A transformação das relações de trabalho e a própria evolução da tecnologia passaram a exigir a reformulação dos cursos e o estabelecimento de uma nova relação ensino - aprendizagem (Haguenauer, Lopez, Martins, Grandão e Filho, 2003). O ensino a distância, é cada vez mais utilizado por escolas, universidades e empresas. Neste contexto, a avaliação dos programas e ferramentas, surge como uma necessidade básica de se conhecer as opções disponíveis, as técnicas e as metodologias mais adequadas para a utilização dos materiais educativos e de se estudar os processos cognitivos relacionados com o uso destes materiais. É importante destacar que a simples incorporação de novas tecnologias, sem as necessárias transformações no processo pedagógico, não conduz a uma verdadeira melhoria na qualidade de ensino. A problemática da avaliação dos ambientes de aprendizagem (online ou não), não é nova e tem sofrido várias abordagens ao longo dos anos. Numa tentativa para sistematizar as contribuições de Carrol (1963), Reeves e Reeves (1997) e Taylor e Maor (2000), Clayton (2004) propôs que se considerem 5 dimensões críticas na análise de ambientes online de aprendizagem:

- Interacção entre o Estudante e a Interface, isto é, quais são as características da interface criados que aumentar/inibir a aprendizagem do aluno e navegação;

- Interacção entre Estudantes, ou seja, como, porquê e quando comunicam os alunos uns com os outros e qual é a natureza dessa comunicação;

- Interacção entre o Tutor e o Estudante, isto é, como, porquê e quando comunicam o alunos com seu tutor e qual é a natureza dessa comunicação;

- Interacção entre o Estudante e os Conteúdos Multimédia, ou seja, como é que o estudante se relaciona e interpreta informações disponibilizadas online;

- Actividades de Reflexão do Estudante, isto é como são os alunos incentivados a reflectir sobre a sua aprendizagem. 
Tendo em consideração estas dimensões, procedeu-se a um estudo prévio com o qual se pretendia identificar, caracterizar e avaliar instrumentos de análise e avaliação de AVA já objecto de processo de validação estatística, tendo-se seleccionado o Web Based Learning Environment Inventory (WEBLEI) para tradução, adaptação e validação na língua Portuguesa (Jesus et al., 2011).

\section{WEB BASED LEARNING ENVIRONMENT INVENTORY (WEBLEI)}

O WEBLEI considera a avaliação de AVA em termos de um conjunto de etapas que compreendem o acesso a materiais, interacção entre os intervenientes, as percepções dos alunos face à aprendizagem na web, e a determinações pessoais face à aprendizagem (Chang e Fisher, 1999, 2001). Estas etapas exprimem-se através de quatro escalas, 1) Acesso (p.e, conveniência, eficiência, autonomia e flexibilidade), 2) Interacção (p.e, a reflexão, interacção, feedback, colaboração), 3) Estruturação e Design (ou seja, objectivos claros, planeamento das actividades, conteúdo apropriado, design, layout, estruturação lógica ) e 4) Resposta, (ou seja, uma escala de atitude relativamente aspectos como, prazer, realização confiança, o sucesso, o tédio, frustração). Considerando as 5 dimensões (ver figura 1) referidas por Clayton (2004), e uma vez que se pretende avaliar o Ambiente Virtual, e não, o estado dos estudantes aquando da instrução, optou-se pela validação e adaptação transcultural de uma versão reduzida do WEBLEI, que contempla 3 escalas - Acesso, Interacção e Estruturação e Design.

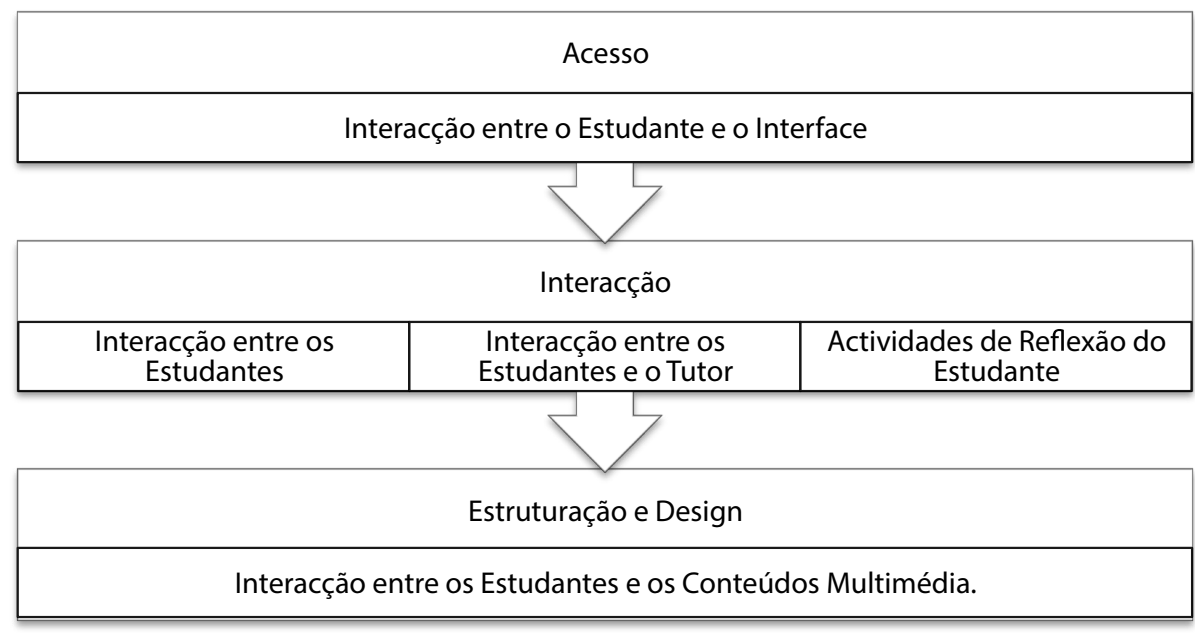

Figura 1. Relação entre as Escalas da versão reduzida do WEBLEI e as dimensões propostas por Clayton (2004) 


\section{ESCALAS E ITENS DE AVALIAÇÃO DO WEBLEI - VERSÃO REDUZIDA}

Além de dados demográficos, na versão reduzida do WEBLEI, existem 3 escalas sendo que as primeiras duas escalas foram adaptadas dos trabalhos de Tobin, (1998) e a escala final centra-se na estruturação e design do ambiente virtual. Este instrumento utiliza uma medida de avaliação de Likert com 5 opções de resposta: "sempre", "frequentemente", "às vezes", "raramente" e "nunca". De seguida apresenta-se cada uma das escalas.

\section{Escala 1: Acesso}

A escala de "Acesso" contempla quatro categorias principais de itens referentes às dimensões: (i) eficiência, (ii) conveniência, (iii) autonomia e (iv) flexibilidade. Segundo os autores:

- "Convenience is achieved when students can access the learning activities at convenient times.

- Efficiency is described as not having to attend on campus classes and therefore allowed for efficient use of time.

- Autonomy is described as allowing students to decide when and how to access the curriculum.

- Flexibility is described as allowing students to meet their goals".

$$
\text { Tobin (1998), citado em Chang e Fisher (1999) }
$$

Os itens seguintes são incluídos na avaliação de Acesso:

- Consegui aceder às actividades e conteúdos, nos momentos que me eram mais convenientes;

- Os conteúdos online e materiais didácticos estavam disponíveis na plataforma, em locais acessíveis;

- Poupei tempo em viagens e na presença nas aulas, para estudar e para outras tarefas;

- Consegui trabalhar ao meu ritmo para atingir os objectivos de aprendizagem propostos; 
- Tive autonomia para decidir a quantidade de conteúdos que queria aceder;

- Tive autonomia para definir quando queria aceder à plataforma;

- A flexibilidade do ambiente virtual permitiu-me atingir os objectivos de aprendizagem;

- A flexibilidade do ambiente virtual permitiu-me explorar as minhas áreas de interesse.

Escala 2: Interacção

Incluído no âmbito desta escala, encontram-se 5 categorias de itens em torno das dimensões: (i) reflexão, (ii) qualidade, (iii) interacção, (iv) feedback e (v) colaboração. Segundo os autores:

- "Reflection is noted as asynchronous interactions which encouraged reflective interactions.

- Quality is linked to the learning reflected in the level of activity undertaken by the students.

- Interaction is described as enabling students to interact with other asynchronously.

- Feedback is described as the availability of feedback from students and the teacher.

- Collaboration enabled students to collaborate in a variety of activities".

Tobin (1998), citado em Chang e Fisher (1999)

Os itens seguintes são incluídos na avaliação da Interacção:

- Pude comunicar de forma electrónica com outros estudantes (via email, fórum, chat...);

- Para que tivesse bons resultados de aprendizagem, tive de criar auto-disciplina; 
- Quando não entendia algum conteúdo ou tarefa, pude questionar o meu tutor/professor;

- Quando não entendia algum conteúdo ou tarefa, pude questionar os meus colegas;

- Quando solicitados, os outros estudantes responderam prontamente às minhas questões;

- Participei regularmente em auto-avaliações;

- Participei regularmente em hetero-avaliações;

- Tive apoio dos meus colegas durante a aprendizagem no ambiente virtual.

Escala 3: Estruturação e Design

Esta escala tem como objectivo, avaliar a estruturação pedagógica e racional do ambiente virtual de aprendizagem, assim como o design do mesmo. Incluído nesta secção estão a relevância e abrangência de conteúdo, validade de conteúdo, facilidade de navegação, layout e aspectos estéticos.

Os itens seguintes são incluídos para a avaliação da Estruturação e Design:

- Os objectivos de aprendizagem estavam estipulados claramente em cada sessão;

- A organização de cada sessão online era perceptível;

- A estruturação das sessões online, permitiu manter-me focado nos respectivos tópicos;

- Os objectivos de cada tarefa/trabalho foram apresentados de forma clara;

- As actividades online foram planeadas cuidadosamente durante o curso;

- Os conteúdos das sessões, foram apropriados para um ambiente virtual;

- A apresentação dos conteúdos foi clara; 
- O ambiente de aprendizagem virtual estimulou o meu interesse ao longo de todo o curso.

No presente estudo, reconhecemos a importância da avaliação dos ambientes virtuais de aprendizagem, como ferramenta essencial para o contínuo processo de melhoria. Com a tradução, adaptação e validação do WEBLEI espera-se obter uma ferramenta quantitativa, fiável e de uso intuitivo.

\section{METODOLOGIA}

\section{Tradução e Adaptação}

A tradução das 3 escalas do WEBLEI, seguiu a metodologia proposta por Almeida e Freire (2008). Após a autorização dos autores do WEBLEI, para a tradução, adaptação e validação para a língua e população portuguesa, procedeu-se à tradução do Inglês para Português de forma independente por dois portugueses bilingues (tradutores). $\mathrm{O}$ investigador também procedeu à tradução independente do instrumento. Durante o período de tradução foram realizados alguns contactos com os tradutores a fim de esclarecer questões relacionadas com a equivalência da tradução do item, ou seja, se a tradução mantém o mesmo significado da versão original (equivalência do item). Foi-lhes explicado o objectivo do instrumento de medida e as intenções subjacentes à concepção de cada item. A análise das diferenças entre as três versões das traduções foi realizada pelo investigador, primeiro autor deste texto, resultando desta forma a primeira versão preliminar do WEBLEI em Português (WEBLEI-01). O passo seguinte consistiu na reflexão falada ("thinking aloud") sobre o instrumento de medida com um grupo com características semelhantes à população em estudo constituído por 15 estudantes de licenciatura. Segundo Almeida e Freire (2008) citando Goldman (1971) uso do método da reflexão falada ou "thinking aloud" é incluído num método de análise qualitativa de itens, quando o objectivo é realizar análises e apreciações quanto ao conteúdo e à forma dos itens, nomeadamente a clareza do item, a sua compreensibilidade e adequação aos objectivos. Considerando as sugestões definidas pelo grupo de "thinking aloud", procedeu-se à redefinição de alguns itens, dando origem à versão WEBLEI-02. Seguidamente a esta fase, deu-se início ao processo de retro tradução. A mesma foi realizada por dois tradutores bilingues, sem conhecimento prévio da escala original, ambos tradutores profissionais (WEBLEI-03). Após consenso na retro tradução, a terceira versão preliminar do WEBLEI foi avaliada por um nativo da língua inglesa e especialista da área da tradução, não havendo alterações significativas a registar.

\section{Amostra}


Um dos aspectos fundamentais da validação transcultural de instrumentos de medida é seguir os mesmos passos do autor original. Tendo por base esta premissa a amostragem foi efectuada tendo presente os mesmos critérios (critérios de inclusão) do estudo original (Chang e Fisher, 2001), ou seja, ser estudante do ensino superior, ter efectuado formação a distância, utilizando ambientes online de aprendizagem, falar fluentemente português, saber ler e escrever, ter idade superior a 18 anos, estar consciente da participação no preenchimento, questionário e em condição física capaz de o preencher. A representatividade de uma amostra é provavelmente a condição mais importante numa investigação, nomeadamente quando se pretende generalizar os resultados obtidos com uma amostra à população. Segundo Almeida e Freire (2008) é fundamental que a amostra seja suficientemente grande, de forma a garantir que numa segunda análise se mantenham os mesmos factores. Contudo existem diferentes orientações no que diz respeito à definição do tamanho da amostra. Pestana e Gageiro (2003) consideram que o mínimo de respostas válidas (N) é obtido pela fórmula $\mathrm{N}=5 \times \mathrm{K}$ (se $\mathrm{K}>15$ ), em que $\mathrm{K}$ é o número de questões do instrumento, logo seriam necessários pelo menos 120 respondentes. Já Almeida e Freire (2008) defendem um critério mais rigoroso, considerando a necessidade de uma amostra de 300 sujeitos, de modo a ser significativa para a validação de instrumentos com um número elevado de itens.

\section{Procedimento de aplicação}

Foi efectuado um levantamento prévio sobre as instituições de ensino superior em Portugal que ofereciam formações conducentes a grau em modalidade b/e-learning. Procedeu-se de seguida a um contacto formal com cada instituição, explicitando os objectivos da investigação, assegurando o anonimato no tratamento global dos dados e pedindo a divulgação juntos dos estudantes. O questionário foi construído na plataforma SurveyMonkey ${ }^{\circledR}$ e disponibilizado através de um URL. Note-se que, no sentido de melhor caracterizar a amostra quanto à origem académica, assim como ao tipo de LMS utilizada, foram adicionadas duas novas questões ao inventário. Mil e trinta e um indivíduos acederam ao questionário, sendo que 694 completaram o preenchimento da primeira escala, 660 completaram o preenchimento de duas escalas e 577 indivíduos, oriundos de diversas instituições de ensino superior portuguesas, preencheram a totalidade do questionário. Em qualquer um dos casos, a amostra foi superior aos 300 indivíduos, estipulados por Almeida e Freire (2008).

\section{RESULTADOS}


Considerando apenas os indivíduos que responderam integralmente ao questionário $(n=577)$ pode verificar-se pela tabela 1 que $68,28 \%$ dos inquiridos são do sexo feminino e que a amostra se distribui entre várias faixas etárias.

\begin{tabular}{|l|l|l|}
\hline Género & Frequência & Percentagem \\
\hline Masculino & 183 & $31,72 \%$ \\
\hline Feminino & 394 & $68,28 \%$ \\
\hline
\end{tabular}

\begin{tabular}{|l|l|l|}
\hline Idade & Frequência & Percentagem \\
\hline $18-20$ & 84 & $14,6 \%$ \\
\hline $21-23$ & 83 & $14,4 \%$ \\
\hline $24-26$ & 55 & $9,5 \%$ \\
\hline $27-29$ & 51 & $8,8 \%$ \\
\hline $30-32$ & 52 & $9,0 \%$ \\
\hline $33-35$ & 47 & $8,1 \%$ \\
\hline $36-38$ & 39 & $6,8 \%$ \\
\hline $39-41$ & 36 & $6,2 \%$ \\
\hline $42-44$ & 38 & $6,6 \%$ \\
\hline $45-47$ & 30 & $5,2 \%$ \\
\hline $48-50$ & 13 & $2,3 \%$ \\
\hline Superior a 50 & 49 & $8,5 \%$ \\
\hline & & \\
\hline
\end{tabular}

Tabela 1. Informações Sócio-Demográficas da Amostra

\begin{tabular}{|l|l|l|}
\hline Sistema de Gestão de Aprendizagem Utilizado & Frequência & Percentagem \\
\hline Moodle & 459 & $79,55 \%$ \\
\hline Blackboard $^{\circledR}$ & 66 & $11,44 \%$ \\
\hline Sakai $^{\circledR}$ & 5 & $0,87 \%$ \\
\hline Dokeos $^{\circledR}$ & 1 & $0,17 \%$ \\
\hline Outro & 46 & $7,97 \%$ \\
\hline
\end{tabular}

Tabela 2. Sistema de Gestão de Aprendizagem utilizado pela amostra

Quanto ao LMS (Sistema de Gestão da Aprendizagem) utilizado durante a aprendizagem (tabela 2), 79,55\% dos inquiridos destacam o uso do Moodle, seguindo a utilização do BlackBoard ${ }^{\circledR}$ (11,44\%). Outros LMS apresentam uma utilização residual de $9,01 \%$. 
Relativamente à instituição de origem (tabela 3), constata-se que 31,01\% dos inquiridos são oriundos da Universidade Aberta, 10,4\% da Universidade de Aveiro, 7,28\% do Instituto Politécnico de Coimbra, 6,93\% do Instituto Politécnico de Viana do Castelo e 5,20\% da Universidade da Beira Interior. As restantes instituições de ensino superior público contemplam 32,93\% dos participantes, sendo que são oriundos de outras instituições de ensino superior, 6,93\% dos inquiridos.

\begin{tabular}{|l|l|l|}
\hline Instituição de Origem & Frequência & Percentagem \\
\hline Universidade Aberta & 179 & $31,02 \%$ \\
\hline Universidade de Aveiro & 60 & $10,40 \%$ \\
\hline Instituto Politécnico de Coimbra & 42 & $7,28 \%$ \\
\hline Outra Instituição de Ensino Superior & 40 & $6,93 \%$ \\
\hline Instituto Politécnico de Viana do Castelo & 36 & $6,24 \%$ \\
\hline Universidade da Beira Interior & 30 & $5,20 \%$ \\
\hline Instituto Politécnico de Setúbal & 29 & $5,03 \%$ \\
\hline Universidade da Madeira & 22 & $3,81 \%$ \\
\hline Universidade Nova de Lisboa & 22 & $3,81 \%$ \\
\hline Instituto Politécnico de Leiria & 21 & $3,64 \%$ \\
\hline Instituto Politécnico de Bragança & 18 & $3,12 \%$ \\
\hline Universidade do Minho & 17 & $2,95 \%$ \\
\hline Instituto Politécnico de Santarém & 14 & $2,43 \%$ \\
\hline Universidade do Algarve & 11 & $1,91 \%$ \\
\hline Instituto Politécnico do Porto & 9 & $1,56 \%$ \\
\hline Instituto Politécnico de Cávado e Ave & 6 & $1,04 \%$ \\
\hline Universidade de Lisboa & 6 & $1,04 \%$ \\
\hline Universidade de Évora & 4 & $0,69 \%$ \\
\hline Instituto Politécnico de Tomar & 4 & $0,69 \%$ \\
\hline Instituto Politécnico da Guarda & 2 & $0,35 \%$ \\
\hline Instituto Politécnico de Lisboa & 2 & $0,35 \%$ \\
\hline Universidade Técnica de Lisboa & 1 & $0,17 \%$ \\
\hline Instituto Politécnico de Castelo Branco & 1 & $0,17 \%$ \\
\hline Instituto Politécnico de Viseu & $0,17 \%$ \\
\hline Un & 3 & \\
\hline
\end{tabular}

Tabela 3. Instituição de origem dos elementos da amostra

\section{Fidelidade}


O estudo da fidelidade (Tabela 4) foi realizado através da determinação do coeficiente Alfa de Cronbach. Uma boa consistência interna deve exceder um $\alpha$ de 0,80 mas são aceitáveis valores acima de 0,60 quando as escalas têm um número muito baixo de itens (Hill e Hill, 2008; Ribeiro, 1999). Uma vez que as três escalas do WEBLEI são independente, o cálculo do coeficiente Alfa de Cronbach foi efectuado tendo por base os números indivíduos que responderam a todas as questões de cada escala. Os valores do alfa de Cronbach obtido são abonatórios de uma boa consistência interna para as três escalas do WEBLEI. A escala de Acesso apresenta um alfa de Cronbach o,884 para o total da escala e o,860 é o valor mais baixo para os itens; a escala de Interacção apresenta um alfa de Cronbach de o,807 para o total da escala e 0,785 é tido como valor mais baixo para os itens e por último a escala de Estruturação e Design apresenta um alfa de Cronbach de 0,941 para o total da escala e 0,932 é o valor mais baixo para os itens. Verifica-se ainda que, na totalidade dos casos, o a desce quando os itens são excluídos, o que significa que quando estão presentes melhoram a homogeneidade da escala. Os valores obtidos indicam uma boa consistência interna no processo de tradução adaptação e validação da versão reduzida do WEBLEI para Português, sendo inclusivamente superiores aos relatados no estudo original (Chang e Fisher, 2001). Ao realizar-se a inspecção da correlação de cada item com o total da escala, verificou-se que os valores oscilam entre 0,438 e 0,817 . De salientar que a correlação do item com o valor encontrado se reporta à correlação do item com a soma dos restantes itens, ou seja, ele próprio foi excluído da soma da escala. Todos os items cumprem o critério de correlação superior a 0,2 (Fortin, 2009).

\section{Validade}

A validade de construto permite-nos saber se é possível encontrarmos um ou mais construtos teóricos das variáveis que a escala pretende avaliar (Anastasi, 1988 citado em Sani, 2004). Através da análise factorial procura-se verificar a validade interna do instrumento, tentando encontrar uma explicação para a variância dos resultados, recorrendo-se para tal à análise de componentes principais (Sani, 2004). Um dos critérios de adequação para a realização da análise de componentes principais foi dado pelo teste de esfericidade de Bartlett (Pestana e Gageiro, 2003). O teste de Bartlett foi significativo $(p<0.000)$, demonstrando que a matriz de correlações é distinta de uma matriz de identidade (Pestana e Gageiro, 2003). Este resultado, em conjunto com o índice de Kaiser-Meyer-Olkin (cujo valor pode ser classificado de muito bom, de acordo com (Hutcheson e Sofroniou, 1999: KMO = 0.936), confirma a factoriabilidade dos dados (Pestana e Gageiro, 2003). A análise factorial foi efectuada atendendo ao critério de decisão de Guttman-Kaiser (valores próprios >1.0) e 
revelou a existência de 5 factores. A análise em componentes principais foi seguida de rotação varimax, um método usado quando se pretende obter uma estrutura simples, uma vez que se trata de uma técnica que maximiza as saturações mais elevadas e reduz a saturações mais baixas. Neste caso e considerando os construtos teóricos do questionário original - critério à priori (Hair, Black, Anderson e Tatham, 2007) - foi forçada uma extracção em 3 factores. Na tabela 5 são apresentados os resultados da análise em componentes principais, seguida de rotação varimax, com a descrição da saturação factorial de cada item nos vários factores, valores próprios, percentagem da variância total e percentagem de variância acumulada explicadas para cada factor, assim como as comunalidades (h2), i.e, a proporção da variância de cada variável explicada pelos componentes principais.

\begin{tabular}{|c|c|c|c|c|c|c|}
\hline Escalas & Items & $\mathrm{N}$ & Média & Variância & $\begin{array}{l}\text { Correlação de } \\
\text { Pearson sem o } \\
\text { item. }\end{array}$ & $\begin{array}{c}\text { Alfa de } \\
\text { Cronbach sem o } \\
\text { item. }\end{array}$ \\
\hline \multirow{8}{*}{ 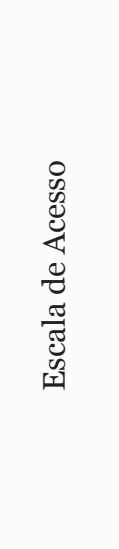 } & Item 1 & 694 & 27,5216 & 26,495 &, 551 & ,879 \\
\hline & Item 2 & 694 & 27,5504 & 26,669 & ,524 & ,881 \\
\hline & Item 3 & 694 & 27,8271 & 22,784 & ,672 & ,868 \\
\hline & Item 4 & 694 & 27,8098 & 23,678 & ,747 &, 860 \\
\hline & Item 5 & 694 & 28,0130 & 22,697 & ,731 & ,861 \\
\hline & Item 6 & 694 & 27,6772 & 23,751 & 697 & ,864 \\
\hline & Item 7 & 694 & 27,8501 & 24,145 & ,703 & ,864 \\
\hline & Item 8 & 694 & 28,0360 & 24,101 & ,607 & 874 \\
\hline
\end{tabular}




\begin{tabular}{|c|c|c|c|c|c|c|}
\hline Escalas & Items & $\mathrm{N}$ & Média & Variância & $\begin{array}{c}\text { Correlação de } \\
\text { Pearson sem o } \\
\text { item. }\end{array}$ & $\begin{array}{c}\text { Alfa de } \\
\text { Cronbach sem o } \\
\text { item. }\end{array}$ \\
\hline \multirow{8}{*}{ 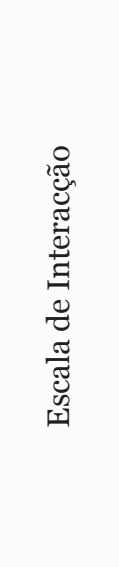 } & Item 9 & 660 & 24,5318 & 32,070 &, 529 & ,785 \\
\hline & Item 10 & 660 & 24,6015 & 32,055 & ,454 & ,799 \\
\hline & Item 11 & 660 & 24,0773 & 34,903 & ,488 & ,791 \\
\hline & Item 12 & 660 & 23,9955 & 33,470 & ,621 & ,775 \\
\hline & Item 13 & 660 & 24,5000 & 33,155 &, 582 &, 778 \\
\hline & Item 14 & 660 & 24,9167 & 32,225 &, 483 & ,792 \\
\hline & Item 15 & 660 & 25,1803 & 32,864 &, 438 & ,800 \\
\hline & Item 16 & 660 & 24,6000 & $31,75^{2}$ & ,664 &, 766 \\
\hline \multirow{8}{*}{ 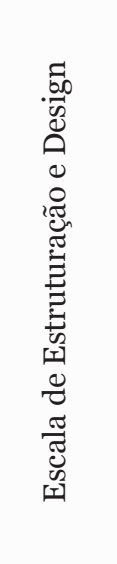 } & Item 17 & 577 & 26,0659 & 29,263 & ,787 & ,934 \\
\hline & Item 18 & 577 & 26,0867 & 29,597 & ,791 & ,933 \\
\hline & Item 19 & 577 & 26,1057 & 29,084 & ,817 & ,932 \\
\hline & Item 20 & 577 & 26,0329 & 30,254 & ,787 & ,934 \\
\hline & Item 21 & 577 & 26,1646 & 29,579 & ,797 & ,933 \\
\hline & Item 22 & 577 & 26,1438 & 29,908 & ,786 & ,934 \\
\hline & Item 23 & 577 & 26,0607 & 29,870 & ,814 & ,932 \\
\hline & Item 24 & 577 & 26,1265 & 29,489 & ,747 & ,937 \\
\hline
\end{tabular}

Tabela 4. Resultados relativos à consistência interna do WEBLEI

Os 3 factores retidos explicam $58,11 \%$ da variância total. Este valor encontra-se acima dos 50\%, considerados necessários por Streiner (1994) e é superior à média de $56,6 \%$ encontrada por Peterson $(2000)^{2}$. É também visível que alguns itens apresentam valores de saturação próximos em diferentes factores, contudo optouse por reter apenas os valores de saturação maiores, uma vez que são estes que se aproximam dos critérios teóricos. 


\begin{tabular}{|c|c|c|c|c|}
\hline \multirow[b]{2}{*}{ N. ${ }^{\circ}$ do item } & \multicolumn{4}{|c|}{ Componentes } \\
\hline & $\begin{array}{c}1 \\
\text { Estruturação e Design }\end{array}$ & $\begin{array}{c}2 \\
\text { Acesso }\end{array}$ & $\begin{array}{c}3 \\
\text { Interacção }\end{array}$ & h2 \\
\hline 17 &, 757 & & & ,719 \\
\hline 18 &, 812 & & & ,728 \\
\hline 19 &, 807 & & & ,755 \\
\hline 20 &, 799 & & &, 713 \\
\hline 21 &, 793 & & &, 725 \\
\hline 22 & ,801 & & & ,709 \\
\hline 23 & ,805 & & &, 746 \\
\hline 24 &, 662 & ,406 & & 678 \\
\hline 1 & &, 467 & & 644 \\
\hline 2 & ,405 & ,435 & &, 618 \\
\hline 3 & &, 733 & & ,645 \\
\hline 4 & &, 777 & & ,695 \\
\hline 5 & &, 836 & &, 736 \\
\hline 6 & &, 736 & & ,621 \\
\hline 7 & & ,692 & & ,625 \\
\hline 8 & &, 614 & & ,488 \\
\hline 9 & & &, 522 &, 548 \\
\hline 10 & & & ,444 & ,617 \\
\hline 11 & & & ,467 &, 585 \\
\hline 12 & & & ,696 &, 729 \\
\hline 13 & & &, 676 & 679 \\
\hline 14 & & &, 627 & ,828 \\
\hline 15 & & & ,599 & ,837 \\
\hline 16 & & &, 716 & ,654 \\
\hline $\begin{array}{l}\text { \% de Variância } \\
\text { Cumulativa }\end{array}$ & 42,157 & 51,028 & 58,116 & \\
\hline
\end{tabular}

Tabela 5. Resultados relativos análise factorial com rotação VARIMAX em 3 factores 


\section{CONSIDERAÇÕES FINAIS}

Tal como referido inicialmente, foram objectivos principais deste trabalho, traduzir, adaptar e aferir a validade e fidelidade de uma versão portuguesa reduzida do WEBLEI. Tendo em conta os resultados apresentados neste artigo, as medidas do WEBLEI parecem reflectir os construtos que pretendem medir - o que constitui um indicador de validade de construto. Os três construtos obtidos, são os esperados conceptualmente e podem ser claramente identificados como Acesso, Interacção e Estruturação e Design. Em termos de consistência interna, foram igualmente encontrados valores satisfatórios para as três escalas. Verificase ainda que, na totalidade dos casos, o a desce quando os itens são excluídos, o que significa que quando estão presentes melhoram a homogeneidade da escala. Também as correlações item-total apresentam valores satisfatórios, contribuindo para uma análise positiva da consistência interna do instrumento. Com base nestes resultados, espera-se que a versão Portuguesa reduzida do WEBLEI, possa constituir um instrumento fiável para a avaliação de ambientes virtuais de aprendizagem.

\section{NOTAS}

1. Embora mais comummente associados à educação a distância, os ambientes online de aprendizagem podem ser amplamente utilizados como suporte à aprendizagem presencial. Sobre este tópico aconselhamos a leitura de Lopes y Gomes (2007).

2. Peterson (2000) realizou um estudo meta-analítico onde avaliou os níveis de variância explicada em estudos que utilizaram Análise Factorial Exploratória. O valor médio encontrado numa amostra de 568 artigos foi de 56,6\% de variância total explicada, porém o autor não sugere pontos de corte para o que seria um nível de variância explicada aceitável ou não aceitável.

\section{REFERÊNCIAS BIBLIOGRÁFICAS}

Almeida, F.; Almeida, M. E. (2003). Educação a distância em meio digital: novos espaços e outros tempos de aprender, ensinar e avaliar. Presented at the Virtual Educa, Miami. [en línea] Disponible en: http://www.educoas.org/webs/ virtualeduca/2003/es/actas/2/2 01 . pdf [consulta 2013, 08 de septiembre].

Almeida, L.; Freire, T. (2008). Metodologia da Investigação em Psicologia e Educação ( $5^{\mathrm{a}}$ ed.). Psiquilibrios.
Ammenwerth, E.; Gräber, S.; Herrmann, G.; Bürkle, T.; König, J. (2003). Evaluation of health information systems-problems and challenges. International journal of medical informatics, 71 (2-3), (125-135).

Carrol, J. (1963). A model of school learning. Teachers College Record, 64, (723-733).

Chang, V.; Fisher, D. (1999). Students' perceptions of the efficacy of Web-based learning environment: the emergence of a new learning instrument. HERDSA 
Annual International Conference. Melbourne, Australia.

Chang, V.; Fisher, D. (2001). The validation and application of a new learning environment instrument to evaluate online learning in higher education. In: Jefrey, P. (Ed.). Presented at the Australian Association for Research in Education Conference, Fremantle, Western Australia: Australian Association for Research in Education.

Clayton, J. (2004). Investigating online learning environments. In: Atkinson, R.; McBeath, C.; Jonas Dwyer, D. e Phillips, R. (Eds.). Beyond the Comfort Zone: Proceedings of the 21st ASCILITE (19720o). Perth, Australia: ASCILITE. [en línea] Disponible en: http://www.ascilite.org.au/conferences/pertho4/procs/ clayton.html [consulta 2013, o8 de septiembre].

Dyson, M.; Campello, B. (2003). Evaluating Virtual Learning Environments: what are we measuring. Electronic Journal of $e$-Learning, 1 (1), (11-20).

Filho, S.; Machado, E. (2002). Aspectos Metodológicos da Avaliação Pedagógica de Ambientes Virtuais de Aprendizagem. Presented at the IX Congresso Internacional de Educação a Distância da ABED.

Fortin, M. F. (2009). Fundamentos e Etapas do Processo de Investigação. Lusodidacta.

Gomes, M. J. (2005). E-learning : reflexões em torno do conceito. In: Freitas, C. V. e Dias, P. (Eds.), Actas do Congresso Internacional sobre Tecnologias da Informação e Comunicação na Educação. Presented at the Challenges 2005. [en línea] Disponible en: http://repositorium. sdum.uminho.pt/handle/1822/2896 [consulta 2013, 08 de septiembre].

Gomes, M. J. (2008). Reflexões sobre a adopção institucional do e-learning : novos desafios, novas oportunidades. $e$-Curriculum, 3 (2).
Haguenauer, C.; Lopez, F.; Martins, F.; Grandão, M.; Filho, F. (2003). Comparative Study of Learning Management Systems.Colabor@-Revista Digitalda CVA, 2 (5).

Hair; Black; Anderson; Tatham. (2007). Analise Multivariada de Dados. Porto Alegre, Brasil: Bookman.

Hill, M.; Hill, A. (2008). Investigação por questionário. Lisboa, Portugal: Silabo.

Hutcheson, G. D.; Sofroniou, N. (1999). The Multivariate Social Scientist: Introductory Statistics Using Generalized Linear Models. SAGE.

Jesus, A.; Cruz, A.; Gomes, M. J. (2011). Online Learning Environment Surveys for Higher Education. Comparative Analysis and Future Research. In VII International Conference of ICT in EducationPerspectives on Innovation. Presented at the Challenges 2011, Braga: Centro de Competência da Universidade do Minho.

Laguardia, J.; Portela, M. C.; Vasconcellos, M. M. (2007). Evaluation in virtual learning environments. Educação e Pesquisa, 33 (3), 513-530. doi:10.1590/S151797022007000300009

Lopes, A.; Gomes, M. J. (2007). Ambientes virtuais de aprendizagem no contexto do ensino presencial: uma abordagem reflexiva. Actas da V Conferência Internacional de Tecnologias de Informação e Comunicação na Educação (814-824). Presented at the Challenges 2007, Braga: Centro de Competência da Universidade do Minho.

Pestana, M. H.; Gageiro, J. N. (2003). Análise de dados para ciências sociais: a complementariedade do SPSS. Sílabo.

Peterson, R. A. (2000). A Meta-Analysis of Variance Accounted for and Factor Loadings in Exploratory Factor Analysis. Marketing Letters, 11 (3), (261-275). doi:10.1023/A:1008191211004

Ramos, F.; Holmes, B.; Leahy, D.; Dolan, D.; Huet, I.; Gardner, J.; Gardner, J. B. 
(2009). Perspectivas e práticas em eLearning no Ensino Superior e no Ensino ao longo da vida em Portugal, na Irlanda e no Reino Unido. Aprendizagem em Ambientes Virtuais (19-52). Porto Alegre, Brasil: Mediação.

Reeves, T. C.; Reeves, P. (1997). Effective Dimensions of Interactive Learning on the World Wide Web. In: Khan, B. (Ed.), Web-based Instruction (59-66). Englewood Cliffs, N.J: Educational Technologies Publications. [en línea] Disponible en: http://citeseerx.ist.psu.edu/ viewdoc/summary?doi=10.1.1.136.5351 [consulta 2013, o8 de septiembre].

Ribeiro, J. L. (1999). Investigação e avaliação em psicologia e saúde. Lisboa, Portugal: Climepsi Editora.

Sani, A. (2004). As crenças, o discurso e a acção : as construções de crianças expostas à violência interparental (Doutoramento). Universidade do Minho, Braga. [en línea] Disponible en: http:// repositorium.sdum.uminho.pt/handle/1822/6958 [consulta 2013, o8 de septiembre].

Siragusa, L.; Dixon, K.; Dixon, R. (2007). Designing quality e-learning environments in higher education. In Proceedings $A S$ CILITE. Presented at the ICT: Providing choices for learners and learning. P, Singapore. [en línea] Disponible en: http:// www.ascilite.org.au/conferences/singaporeo7/procs/siragusa.pdf [consulta 2013, o8 de septiembre].

Streiner, D. L. (1994). Figuring out factors: the use and misuse of factor analysis. Canadian journal of psychiatry. Revue canadienne de psychiatrie, 39 (3), (135140).

Taylor, P.; Maor, D. (2000). Assessing the efficacy of online teaching with the Constructivist online learning environment survey. Presented at the 9th
Annual Teaching Learning Forum, Perth, Australia. [en línea] Disponible en: http://researchrepository.murdoch. edu.au/8750/ [consulta 2013, o8 de septiembre].

Tobin, K. (1998). Qualitative and quantitative landscapes of classroom learning environments. Learning Environments Research, 1, (139-162).

Tobin, K.; Fraser, B. (1998). Qualitative and quantitative landscapes of classroom learning environments. In Tobin, K. G.; Fraser, B. J. (Eds.), The International Handbook of Science Education. Dordrecht, The Netherlands: Kluwer.

Valcke, M.; Leeuw, F. (2000). Evaluating digital distance learning programs and activities: studies, practices, and recommendations. [en línea] Disponible en: http://agris.fao.org/agrissearch/search/display.do?f=2012/US/ US2012417000170.xml;US2012417007 [consulta 2013, o8 de septiembre].

Valentini, C.; Soares, E. (2010). Fluxos de interação: uma experiência com ambiente de aprendizagem na Web. In Aprendizagem, Ambientes Virtuais, Compartilhando Ideias e Construindo Cenários. ( $2^{\mathrm{a}}$ ed.). Caxias do Sul. [en línea] Disponible en: http://www.ucs.br/etc/revistas/index.php/aprendizagem-ambientes-virtuais/article/viewFile/393/323 [consulta 2013, 08 de septiembre].

Vavassori, F.; Raabe (2003). Organização de atividades de aprendizagem utilizando ambientes virtuais: um estudo de caso. In: Silva, M. (Ed.), Educação Online Teorias, Práticas, Legislação e Formação Corporativa. São Paulo: Loyola. [en línea] Disponible en: http://www. saladeaulainterativa.pro.br/livro educonline apresentacao.htm [consulta 2013, o8 de septiembre]. 


\section{PERFIL PROFISSIONAL E ACADÉMICO DOS AUTORES}

Ângelo Jesus. Licenciado em Farmácia e Doutorando em Ciências da Educação na especialidade de Tecnologia Educativa. É docente da Escola Superior de Tecnologia da Saúde do Porto, desde 2009, onde lecciona disciplinas de Farmacologia e Terapêutica. Desenvolve investigação no âmbito do Case Based Learning Digital, Desenho Instrucional, e desenvolvimento de objectos de aprendizagem para ensino das Ciências da Saúde. Actualmente é membro na Unidade de e-learning do Instituto Politécnico do Porto.

Email: acj@eu.ipp.pt

Maria João Gomes. Professora auxiliar no Departamento de Estudos Curriculares e Tecnologia Educativa, Instituto de Educação da Universidade do Minho - Braga, Portugal. Doutorada em Educação, com especialização em Tecnologia Educacional, já ministrou vários cursos de graduação e pós-graduação em educação a distância e e-learning, tecnologia educacional, didáctica da informática, TIC para alunos com necessidades especiais e jogos digitais e educação. É a autora de mais de 70 publicações e desenvolve os seus interesses de pesquisa em torno dos temas da web social, portfolios digitais, cyberbullying, segurança na Internet, educação a distância e e-learning. É igualmente directora da revista electrónica "Educação, Formação \& Tecnologias" e coordenadora do Centro de Competência de Tecnologias da Informação e Comunicação da Universidade do Minho.

Email: mjgomes@ie.uminho.pt

Agostinho Cunha. Licenciado em Farmácia e pós-graduado em Epidemiologia e Saúde Pública. É docente da Escola Superior de Tecnologia da Saúde do Porto, onde lecciona disciplinas de Microbiologia, Investigação em Saúde, Farmacologia e Bioestatística.

Email: afc@estsp.ipp.pt

Agostinho Cruz. Licenciado em Farmácia e Biologia, Mestre em Ecologia Aplicada e Doutorado em Ciências Biomédicas. Actualmente é presidente da Escola Superior de Tecnologia da Saúde do Porto, onde promove o desenvolvimento de actividades e formações a distância.

Email: asc@estsp.ipp.pt 


\section{DIRECCIÓN DE LOS AUTORES:}

Escola Superior de Tecnologia da Saúde do Porto.

Instituto Politécnico do Porto

Rua Valente Perfeito, $\mathrm{n}^{\mathrm{O}} 322$

4400 - 303 Vila Nova de Gaia

Portugal

Fecha de recepción: 07/05/2013

Fecha de aceptación: 08/09/2013

\section{Como citar este artículo:}

Jesus, A.; Gomes, M. J.; Cunha, A. Cruz, A. (2014). Validade e fidelidade da versão portuguesa reduzida do web based learning environment inventory. RIED. Revista Iberoamericana de Educación a Distancia, volumen 17, nº 1, pp. 179-199. 